\title{
Activating cGAS-STING pathway for the optimal effect of cancer immunotherapy
}

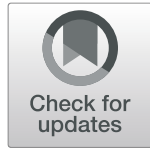

Anping $\mathrm{Li}^{1+}$, Ming $\mathrm{Yi}^{2 \dagger}$, Shuang $\mathrm{Qin}^{2}$, Yongping Song ${ }^{3}$, Qian $\mathrm{Chu}^{2}$ and Kongming $\mathrm{Wu}^{1,2^{*}}$ (D)

\begin{abstract}
During tumor progression, a subset of cancer cells escape from immune surveillance and eventually develop into measurable tumor mass. Cancer immunotherapy eradicates tumor cells by enhancing multiple steps in cancerimmunity cycle including antigen presentation, T cell priming, activation, and immune killing activity. Immunotherapy has been verified as an effective strategy in multiple cancers, but some problems still exist in actual clinical practice such as frequent primary and adaptive resistance. Combination with other adjuvant therapies gives us a new perspective to overcome the emerging obstacles in immunotherapy application. Recently, a series of studies demonstrated that the vital component of host innate immunity — cGAS-STING pathway might play an important role in anti-cancer immunity. It is generally acknowledged that the downstream signals of CGAS-STING especially type I interferon (IFN) bridge innate immunity and adaptive immunity. Given the functions of type I IFN in promoting the maturation and migration of dendritic cells, enhancing cytotoxic T lymphocyte- or natural killer cell-mediated cytotoxicity effect, and protecting effector cells from apoptosis, we believe cGAS-STING agonist might be used as sensitizer for multiple immunotherapies such as cancer vaccine, immune checkpoint blockade, and chimeric antigen receptor T cell therapy. In this review, we highlight the latest understanding of cGAS-STING pathway and the advances of the combination therapy of STING agonist and immunotherapy.
\end{abstract}

Keywords: CGAS-STING, Innate immunity, Type I interferon, Cancer immunotherapy, CAR-T, Immune checkpoint inhibitor

\section{Introduction}

Accumulating mutations in cancer cells not only render malignant transformation, but also activate host's anti-tumor immune response. However, under selective pressure, cancer cells with high immunogenicity are eliminated while ones with low immunogenicity survive. This process is called immunoediting [1]. Dynamically evolving antigen spectrum endows cancer with the capability of immune escape [2]. Apart from immunoediting, other factors such as immunosuppressive tumor microenvironment contribute to immune evasion as well [3]. Cancer immunotherapy is developed to counteract multiple inhibitory immune factors, from impaired cancer antigen presentation to unleashed cancer-killing activity [4-6]. During past few decades, multiple cancer immunotherapies have been

\footnotetext{
* Correspondence: wukm@tjh.tjmu.edu.cn; wukm_lab@163.com Anping Li and Ming Yi are equal contributors.

'Department of Oncology, The First Affiliated Hospital of Zhengzhou University, Zhengzhou 450052, China

${ }^{2}$ Department of Oncology, Tongji Hospital of Tongji Medical College, Huazhong University of Science and Technology, Wuhan 430030, China Full list of author information is available at the end of the article
}

successfully applied in clinical practice including oncolytic virus, chimeric antigen receptor $\mathrm{T}$ cell $(\mathrm{CAR}-\mathrm{T})$, bispecific antibody, and immune checkpoint inhibitor (ICI) [7-10]. Most immunotherapies are aiming to enhance adaptive anti-tumor immunity.

Actually, adaptive anti-tumor immunity is highly dependent on robust innate immunity [11]. As the first immune barrier of host, innate immunity could sense non-self-material by various pattern recognition receptors (PRRs) such as cytosolic DNA sensor [12]. Malignant transformation usually accompanies formation of cytosolic chromatin fragments and micronuclei, increasing the probability of DNA leakage in cancer cell or cancer cell-derived DNA uptake by dendritic cell (DC) [13]. Stimulated by cytosolic DNA, active cyclic GMP-AMP synthase-stimulator of interferon genes (cGAS-STING) pathway stimulates the expression of type I interferon (IFN) in cancer cells or DCs, initiating innate anti-cancer immunity [13-16]. Actually, type I IFN is a versatile molecule related with cell senescence and inflammation response [17]. It has been verified that type I

(c) The Author(s). 2019 Open Access This article is distributed under the terms of the Creative Commons Attribution 4.0 International License (http://creativecommons.org/licenses/by/4.0/), which permits unrestricted use, distribution, and reproduction in any medium, provided you give appropriate credit to the original author(s) and the source, provide a link to the Creative Commons license, and indicate if changes were made. The Creative Commons Public Domain Dedication waiver (http://creativecommons.org/publicdomain/zero/1.0/) applies to the data made available in this article, unless otherwise stated. 
IFN signal is essential to the cross-priming of the tumor-specific T cells [18].

Since STING molecule was found in 2008 [19, 20], substantial efforts have been expended to find an appropriate cGAS-STING agonist for anti-cancer agent development. Actually, cGAS-STING agonists not only induce cancer cell senescence but enhance adaptive anti-cancer immunity which would synergize with immunotherapies [21-24]. In this review, we highlight the latest understanding and the advances of cGAS-STING-targeting strategies, especially in combination with immunotherapies such as cancer vaccine, ICI, oncolytic virus, and chimeric antigen receptor $\mathrm{T}$ cell $(\mathrm{CAR}-\mathrm{T})$ therapy.

\section{The role of CGAS-STING pathway in anti-cancer immunity cGAS-STING pathway}

STING is a cytosolic DNA sensor anchored in endoplasmic reticulum (ER) [25-27]. STING pathway could not be directly activated by double-stranded DNA (dsDNA). Instead, STING pathway is predominantly activated by second messenger cyclic dinucleotide $(\mathrm{CDN})$ which is generated by cGAS [28]. Cytosolic dsDNA directly binds to cGAS and subsequently catalyzes the production of cyclic GMP-AMP (cGAMP) (Fig. 1a) [29, 30]. Following the stimulation of cGAMP, the conformation of STING molecule is changed from monomer to dimers (Fig. 1b) [13]. Then, STING dimers are translocated to perinuclear microsome from ER via Golgi apparatus [13]. STING could recruit and activate TANK-binding kinase 1 (TBK1) which further phosphorylates interferon regulatory transcription factor 3 (IRF3) and upregulates the expression of type I IFN [31]. In addition, STING could activate nuclear factor kappa-light-chain-enhancer of activated B cells (NF-KB) pathway by binding to IкB kinase (IKK) and NF-kB-inducing kinase (NIK) [32, 33]. Activated NF- $\mathrm{KB}$ pathway collaborates with TBK1-IRF3 pathway to induce the expression of type I IFN (Fig. 1c) [13]. Type I IFN has multiple immune-stimulatory functions promoting the maturation, migration, and activation of multiple immune cells such as DC, T cell, and natural killer cell (NK) [18].

Apart from recognizing foreign DNA, cGAS-STING pathway could sense self-DNA derived from damaged and dying cells, which contributes to sterile inflammation in the context of autoimmune diseases and anti-cancer immunity [18]. It is generally believed that cancer-derived DNA could get in DC cytoplasm and stimulate cGAS-STING-type I IFN pathway, which is essential to subsequent activation of T cell [18]. Notably, the biological effect of cGAMP-STING pathway is not limited in a single cell. Both extracellular type I IFN and cell-cell transferred cGAMP could induce regional immune response [34].

As mentioned above, cGAS surveillance occurs in cytoplasm. However, the function of cGAS changes along with its subcellular location [35]. Distinguished from cytoplasmic cGAS, nuclear cGAS could interfere with the formation of PARP-Timeless complex and impede homologous recombination [35]. Nuclear shuttle of cGAS is induced by DNA damage, eventually leading to genome instability and malignant transformation [35]. Therefore, intracellular regulators of cGAS translocation such as B-lymphoid tyrosine kinase and karyopherin2 would be predictive biomarkers and treatment targets for a subset of cancer patients [35].

\section{cGAS-STING pathway in cancer cell}

For normal eukaryotic cell, DNA is strictly separated from cytoplasm to avoid auto-inflammation [36]. However, in tumor cell, the probability of exposure of DNA to cytosolic DNA sensor increases [37-39]. Even though the mechanisms by which nuclear DNA leaks into cytoplasm have not been completely understood, some factors are speculated to contribute to initiate endogenous DNA sensing [40]. Among these factors, the frequent formation of micronuclei plays a vital role in cGAS surveillance [40]. Due to the characteristic of genome instability, cancer cells usually undergo chromosome mis-segregation during cell division [41, 42]. The lagging chromosomes are enveloped by original nuclear membrane which further forms micronuclei [40, 43]. Micronuclei membrane is easy to break down and results in the release of contained dsDNA [44]. Besides micronuclei, small DNA fragments derived from DNA damage could be released into cytoplasm during the whole interphase which could activate cGAS-STING pathway as well [45-48]. In the context of reactive oxygen species (ROS) response, mitochondrial DNA leakage is another contributing factor to stimulating cytosolic DNA sensor and activating STING signaling [49].

The results of co-culture of cancer cells and effector cells showed that cancer cells with downregulated cGAS-STING pathway could resist to immune killing [50]. Further study in mouse model demonstrated that downregulated cGAS-STING pathway led to decreased tumor-infiltrating $\mathrm{CD}^{+} \mathrm{CD}^{+} \mathrm{T}$ cells by reducing the expression of downstream genes of type I IFN such as chemokine (C-X-C motif) ligands 9 and 10 (CXCL9 and CXCL10) [50]. Independent of enhanced anti-cancer immunity, cGAS-STING pathway could directly activate senescence and apoptosis signaling pathways in cancer cells $[51,52]$. cGAS-STING pathway downregulates the expression of anti-apoptosis protein $\mathrm{BCL} 2$ and upregulates the abundance of pro-apoptosis protein BCL2-associated X (BAX) [52]. BAX-mediated mitochondrial outer membrane permeabilization and simultaneous caspase-9-driven caspase-3 activation contribute to cell apoptosis [53]. Therefore, intact cGAS-STING pathway is an important regulator of cancer cell growth, senescence, and immune surveillance. As the consequence of selective 
A

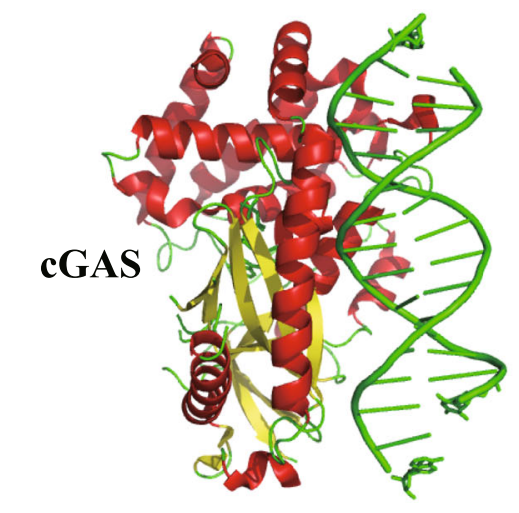

B

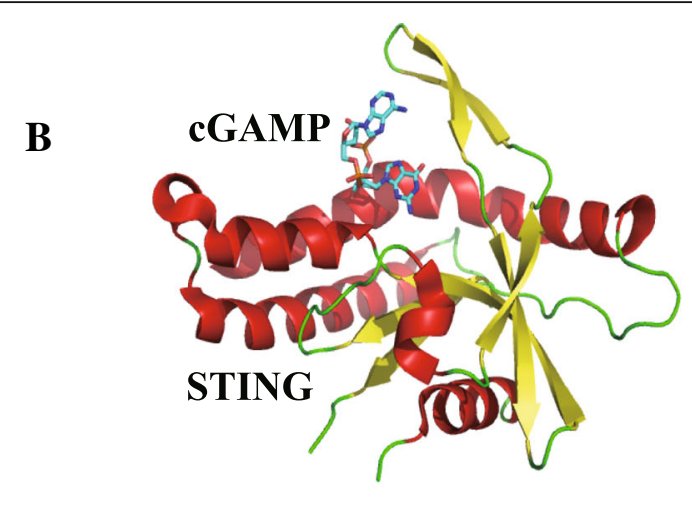

C

dsDNA

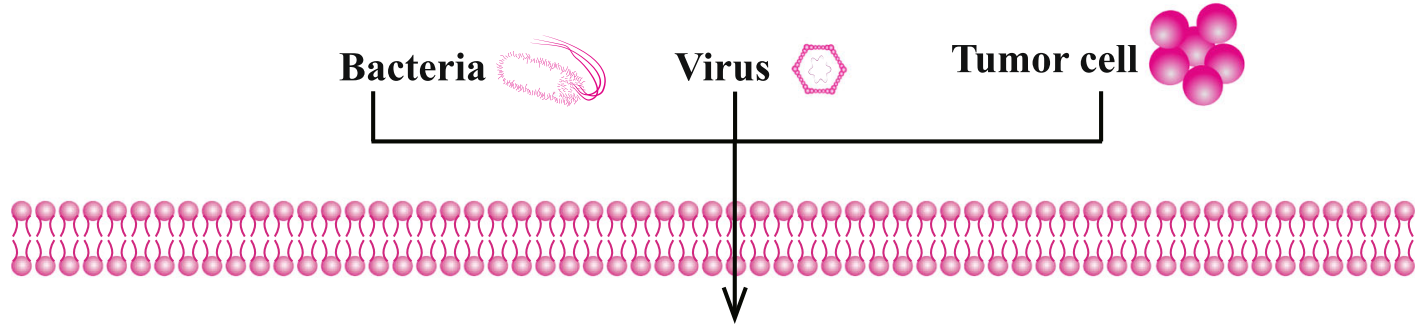
dsDNA NoOOC
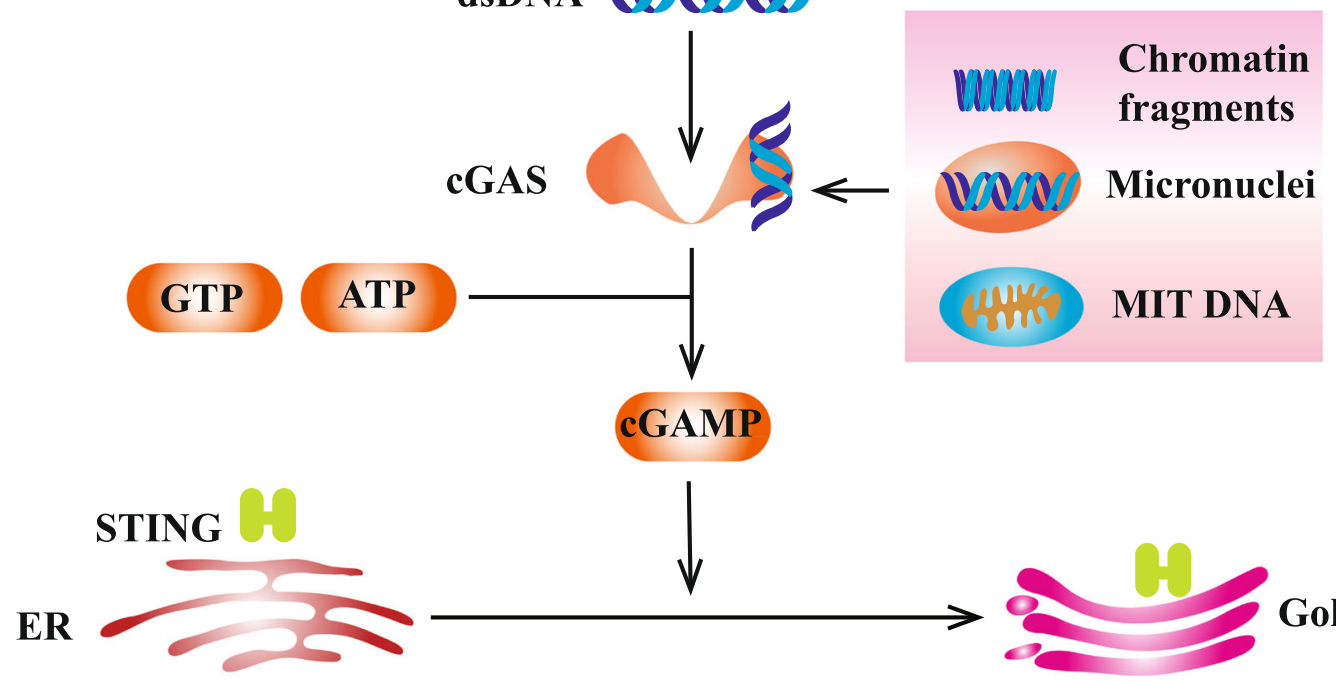
Golgi
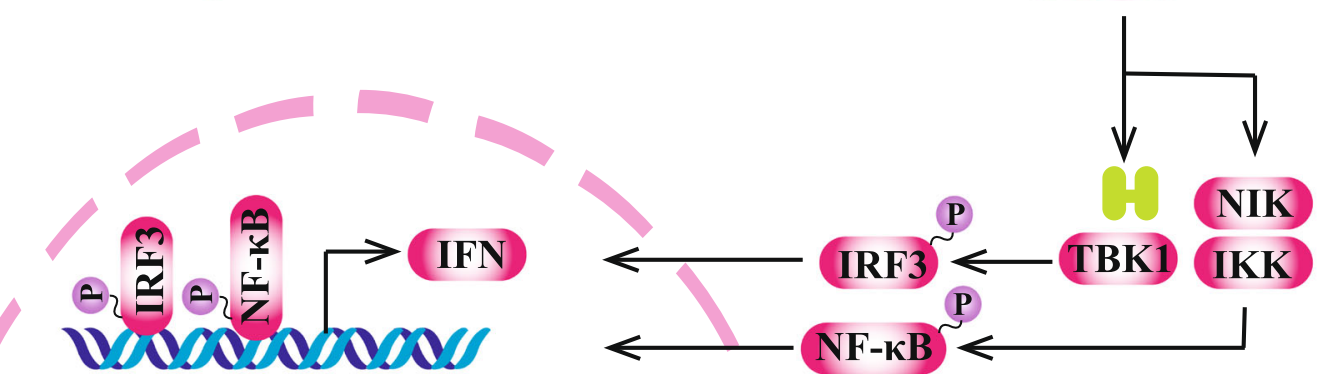

Fig. 1 (See legend on next page.) 
(See figure on previous page.)

Fig. 1 cGAS-STING pathway. a Three-dimensional structure of cGAS-dsDNA complex. b Three-dimensional structure of STING. The above structures were presented by PyMOL Molecular Graphics System. c CGAS-STING pathway. Cytosolic DNA sensor could be activated by exogenous DNA derived from bacteria, virus, or damaged/dying tumor cell. In addition, cGAS could sense endogenous chromosomal fragments, as well as DNA leakage from micronuclei and mitochondria. CGAS directly binds to tumor-derived dsDNA and subsequently catalyzes the production of cyclic GMP-AMP (cGAMP). Following the stimulation of CGAMP, the conformation of STING molecule is changed from monomer to dimers. Then, STING dimers are translocated to perinuclear microsome from ER via Golgi apparatus. STING could recruit and activate TANK-binding kinase 1 (TBK1) which further phosphorylates interferon regulatory transcription factor 3 (IRF3) and upregulates the expression of type I IFN. In addition, STING could activate nuclear factor kappa-light-chain-enhancer of activated B cells (NF-KB) pathway by binding to IKB kinase (IKK) and NF-KBinducing kinase (NIK). Activated NF-KB pathway collaborates with TBK1-IRF3 pathway to induce the expression of type I IFN. Abbreviations: CGAMP, cyclic GMP-AMP; ER, endoplasmic reticulum; IKK, IKB kinase; IRF3, interferon regulatory transcription factor 3; MIT, mitochondria; NF-KB, nuclear factor kappa-light-chain-enhancer of activated B cells; NIK, NF-KB-inducing kinase; TBK1, TANK-binding kinase 1

pressure, surviving cancer cells tend to harbor deficiencies in cGAS-STING pathway. It has been detected that the activation of cGAS-STING is usually impaired in multiple cancers by epigenetic hypermethylation [46].

\section{CGAS-STING pathway in DC}

In tumor microenvironment, cGAS-STING in DC plays an important role in the cross-presentation and priming of tumor-specific $\mathrm{CD}^{+} \mathrm{T}$ cell (Fig. 2). Tumor-derived DNA could be taken up by DC like protein antigen, resulting in the following upregulation of type I IFN [54]. Type I IFN contributes to most biological effects of cGAS-STING pathway on immune cells. Firstly, type I IFN reinforces the cross-presentation of DC by promoting antigen retention and $C D 8 \alpha^{+}$DC survival [35]. Besides, DC cultured with type I IFN shows increased expression of CCR7 which indicates improved lymph node-homing capability [55]. In addition, type I IFN upregulates the expression of multiple Th1 chemokines including CXCL9 and CXCL10 which is important for the homing of APC and trafficking of effector $\mathrm{T}$ cell [56]. STING deletion in DC could abrogate the capability of antigen presentation and decrease the abundance of TIL [57]. Even though cGAS-STING pathway could induce the activation of apoptosis pathway in cancer cell, the pro-apoptosis role of cGAS-STING remains to be further determined in immune cells $[58,59]$.

\section{STING status in immune cell}

Activated cGAS-STING signals could be detected in both tumor cells and immune cells. Although STING activation in tumor cells participates in anti-tumor immune response, active cGAS-STING pathway in host immune cells mainly contributes to tumor control [54]. Sivick et al. confirmed that stimulating cGAS-STING pathway in immune cells could effectively initiate anti-tumor inflammation in mouse bearing STING-deficient tumor [60]. On the contrary, by establishing mouse melanoma xenograft model (B16), Fu et al. observed that STING-deficient mice poorly responded to STING-based cancer vaccine compared with wild-type mice [61].

Among immune cells, DC acts as the core of anti-tumor immunity. The STING molecule in DC magnifies the signal from cytosolic DNA sensor and boosts tumor-specific adaptive immunity. On the one hand, DC directly takes up extracellular DNA from damaged or dying tumor cells $[54,62]$. On the other hand, intracellular STING in DC could be activated by extrinsic cGAMP from tumor cell or artificially added analogues [63]. Marcus et al. investigated the influence of extracellular cGAMP in tumor microenvironment in mouse RMA-S lymphoma model [63]. Following cGAMP injection into RMA-S tumor and inhibition of Golgi transport activity, intracellular cytokine staining showed accumulating IFN- $\beta$ in $\mathrm{CD}_{1} 1^{+}$infiltrating lymphocytes [63].

\section{cGAS-STING pathway in immune regulation}

In general, cGAS-STING signaling pathway is favorable to anti-cancer immune response. However, cGAS-STING was also observed as an inhibitory component in cancer immune microenvironment in some studies. In Lewis lung carcinomas, the application of STING agonist could induce immune tolerogenic state by inhibitory signal indoleamine 2,3-dioxygenase (IDO), which protects cancer cell from immune attack and promotes tumor outgrowth [64, 65]. Additionally, after intratumoral injection of high dose of STING agonist, the increased apoptosis markers and decreased cellularity were noticed in tumor-draining lymph node (TDLN) of injected side [60]. In tongue squamous cancer patients, the expression of STING was positively correlated with the abundance of regulatory T cell (Treg) [66].

As a versatile pathway, the role of cGAS-STING needs further investigation especially considering that its function changes along with agonist dose, cancer type, and disease stage [37]. Notably, advances of immunotherapy provide multiple feasible approaches to reprogram tumor immune microenvironment together with cGAS-STING agonist.

\section{The advances of STING agonist Dimethyloxoxanthenyl acetic acid}

A growing body of evidence demonstrated the important role of cGAS-STING pathway in bridging anti-tumor innate immunity and adaptive immunity [17]. Therefore, the pharmacological activation of cGAS-STING pathway 


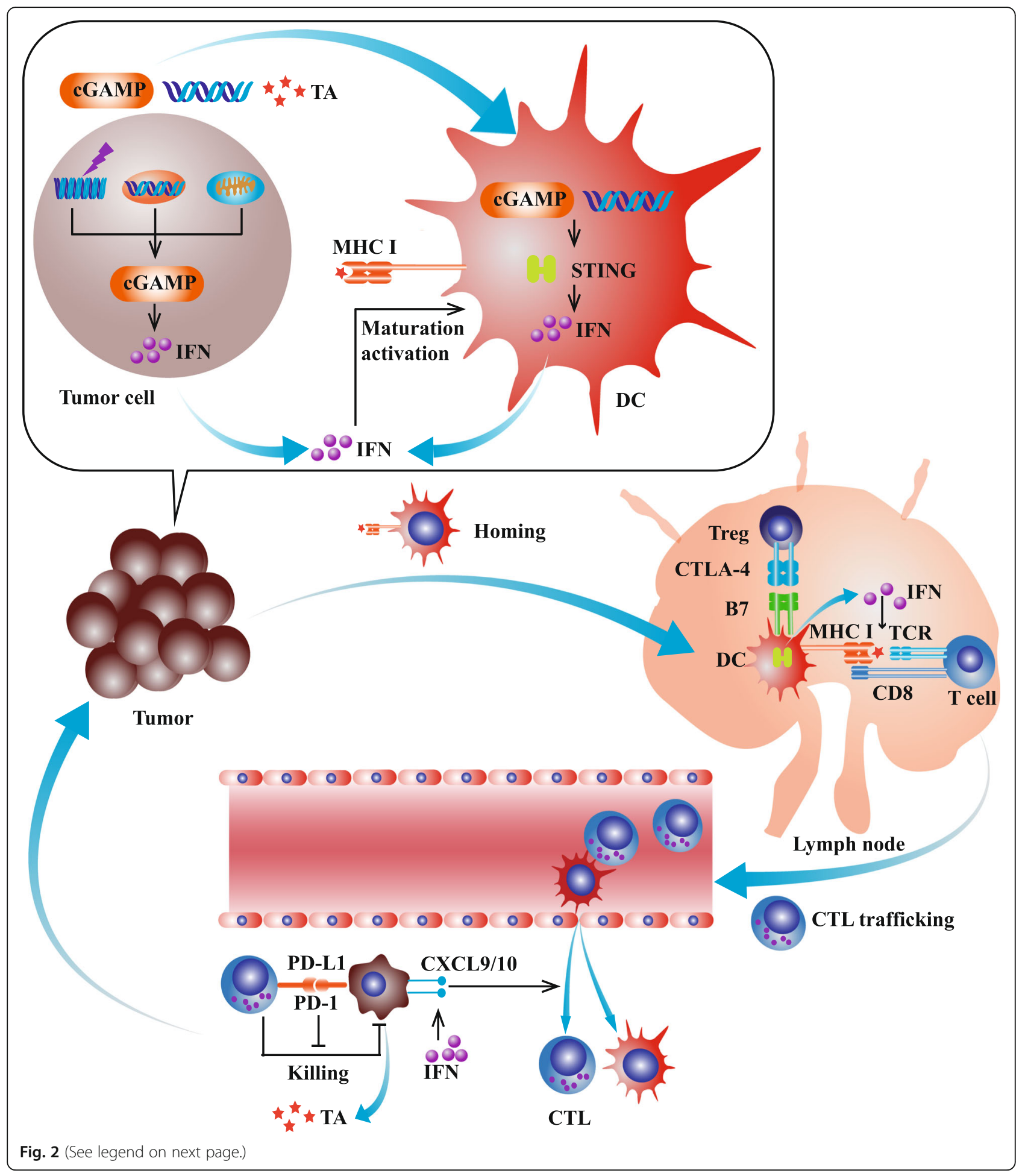


(See figure on previous page.)

Fig. 2 The role of CGAS-STING pathway in anti-tumor immunity. The CGAS-STING pathway upregulates multiples steps in cancer-immunity cycle. DNA leakage not only activates STING pathway in tumor cell, but also promotes STING activation in dendritic cell by DNA uptake or cGAMP transfer. In tumor microenvironment, CGAS-STING in DC plays an important role in the cross-presentation and priming of tumor-specific CD8 ${ }^{+} \mathrm{T}$ cell. Tumor-derived DNA could be taken up by DC like protein antigen, resulting in the following upregulation of type I IFN. Type I IFN contributes to most biological effects of CGAS-STING pathway on immune cells. Firstly, type I IFN reinforces the cross-presentation of DC by promoting antigen retention and CD8a+ DC survival. Besides, DC cultured with type I IFN shows increased expression of CCR7 which indicates improved lymph node-homing capability. In addition, type I IFN upregulates the expression of multiple Th1 chemokines including CXCL9 and CXCL10 which is important for the homing of APC and trafficking of cytotoxic T lymphocytes. Abbreviations: CGAMP, cytosolic GMP-AMP; CTL, cytotoxic T lymphocytes; CTLA-4, cytotoxic T-lymphocyte-associated protein 4; CXCL9, chemokine (C-X-C motif) ligand 9; IFN, interferon; MHC, major histocompatibility complex; PD-1/PD-L1, anti-programmed death-1/programmed death-ligand 1; TA, tumor antigen; TCR, T cell receptor; Treg, regulatory T cell

would be a potential cancer treatment strategy (Table 1). Dimethyloxoxanthenyl acetic acid (DMXAA) is the first agent targeting cGAS-STING pathway [67]. Actually, DMXAA was originally designed as an anti-angiogenesis agent which was latterly found to directly interact with STING [68]. DMXAA showed potent tumor-killing effect in multiple mice models but it failed in phase III clinical trial [68]. Conlon et al. found that the interaction between DMXAA and STING was restricted in mice. In human, this interaction was too weak to induce downstream type I IFN [68].

\section{Cyclic dinucleotides}

Motivated by effective anti-tumor effect of DMXAA in mouse model, researchers have always been trying to find an appropriate human cGAS-STING agonist. It has been verified that both host- and bacteria-derived $C D N$ could activate cGAS-STING pathway in innate immunity. Apart from natural $\mathrm{CDN}$, synthetic $\mathrm{CDN}$ was developed for more robust immune response [26, 28]. Artificially synthetic CDN such as mixed linkage dithio CDN (ML RR-S2 $\mathrm{CDN}$ ) is resistant to snake venom phosphodiesterase and possesses higher binding affinity to all common human STING alleles [69]. ML RR-S2 CDA, also known as ADU-S100 developed by Aduro Biotech, has showed its anti-cancer effect in multiple mouse models $[69,70]$. The in vitro experiments demonstrated that ADU-S100 could promote human peripheral blood mononuclear cell (PBMC) to generate pro-inflammation cytokines such as IFN- $\beta$ [69]. In vivo experiment, Sivick et al. found that the anti-tumor effect of CDN changed along with intratumoral injection dose [60]. In mouse models, high-dose intratumoral injection of ADU-S100 (500 $\mu \mathrm{g})$ could eliminate tumor which might largely depend on non-adaptive immunity fashions such as innate or cytotoxic mechanisms [60]. On the contrary, low-dose intratumoral ADU-S100 mainly activated adaptive anti-tumor immunity [60]. To further explore the efficacy of ADU-S100, two phase I clinical trials are ongoing.

CDNs possess the capability to induce anti-tumor inflammation in theory, but the actual treatment effect of CDNs without appropriate carrier is limited [71]. Due to the characteristics of electronegativity and high water solubility, it is hard for CDNs to cross cellular membrane and activate cytoplasmic STING [71]. Therefore, developing drug carrier with high bioavailability would be meaningful for enhancing therapeutic effect of CDNs [71]. Besides, another challenge for $\mathrm{CDN}$ application is drug delivery. Traditional $\mathrm{CDN}$ delivery by intratumoral injection has two main problems. Firstly, due to the heterogeneity among different tumor lesions even in the same individual, intratumoral injection-induced anti-tumor immunity could not cover all tumor antigen spectrum [60]. Moreover, for some inaccessible tumors, intratumoral delivery of STING agonist is an

Table 1 The anti-cancer effect of CGAS-STING agonist

\begin{tabular}{|c|c|c|c|}
\hline cGAS-STING agonist & Cancer type & Agent delivery & Ref. \\
\hline 3'3'-cGAMP & Mouse B cell malignancies & Intraperitoneal injection & [109] \\
\hline 2'3'-cGAMP & Mouse lymphoma & Intratumoral injection & [63] \\
\hline ML RR-S2 CDG & Mouse melanoma & Intratumoral injection & [69] \\
\hline ML RR-S2 cGAMP & Mouse melanoma & Intratumoral injection & [69] \\
\hline ML RR-S2 CDA (ADU-S100) & Mouse melanoma, colon cancer, mammary carcinoma & Intratumoral injection & {$[61,69,70]$} \\
\hline $\mathrm{DMXAA}^{+}$ & Mouse lung cancer, mesothelioma, human lung cancer, and prostate cancer & Intravenous injection & [110-112] \\
\hline Cyclic di-GMP & Mouse melanoma, prostate cancer, glioma, breast cancer & Intratumoral injection & {$[22,94,113,114]$} \\
\hline DiABZI & Mouse colon tumor & Intravenous injection & {$[72]$} \\
\hline
\end{tabular}

Agent delivery listed in the table is the common delivery approach of cGAS-STING agonist. Cyclic dinucleotide encapsulated by some nanoparticles such as endosomolytic polymersomes could be delivered by intravenous injection as well [71]

cGAMP cyclic GMP-AMP, CDA cyclic di-AMP, ML mixed linkage, DMXAA 5,6-dimethylxanthenone-4-acetic acid, DiABZI dimeric amidobenzimidazole, CDG cyclic di-GMP

${ }^{+}$Mouse STING-specific agonist with weak binding affinity to human STING, failing to pass phase III clinical trials 
unfeasible treatment strategy [72]. Therefore, a novel delivery system or STING agonist which could be systemically delivered would be more valuable for clinical application.

\section{Dimerized amidobenzimidazole}

In 2018, Ramanjulu et al. reported a small molecular STING agonist with systemic anti-cancer effect [72]. This novel STING agonist was designed based on amidobenzimidazole (ABZI) which had modest binding affinity to STING subunit [72]. However, the binding affinity was significantly enhanced after dimerization by 4-carbon butane linker (di-ABZI) [72]. Human PBMC samples were collected to analyze the dose-dependent activation of STING as evaluated by IFN- $\beta$ level [72]. The results showed that concentration for half maximal effect (EC50) of di-ABZI was markedly lower than cGAMP [72]. Mice bearing subcutaneous CT-26 tumor were used to assess the anti-cancer effect of di-ABZI [72]. Mice undergoing di-ABZI treatment had a great advantage in tumor control and survival data over vehicle group [72]. Notably, $80 \%$ of di-ABZI-treated mice kept tumor free until the end of the study [72].

\section{The application of STING agonist in immunotherapy STING agonist: cancer vaccine adjuvant}

Due to central and peripheral tolerance, tumor-associated antigen (TAA) is characterized by weak immunogenicity $[73,74]$. Therefore, an appropriate adjuvant is essential to overcome tolerance and boost tumor-specific immunity. It is confirmed that triggering innate immunity could facilitate the activation of APC, which subsequently enhances pre-existing TAA specific or induces vigorous tumor-specific immunity [73]. In the development of cancer vaccine, multiple adjuvants are widely adopted such as live-attenuated tuberculosis vaccine [75]. When adjuvant and TAA are delivered together, Th1 skewing immune response is initiated [73]. The adjuvant function of CDN has been verified in the development of $\mathrm{H} 5$ influenza vaccine [76]. Given the core role of STING in the initiation of innate immunity, it was speculated that STING stimulator could serve as cancer vaccine adjuvant [27].

In multiple tumor-bearing mice models, Fu et al. firstly investigated the efficacy of STING agonist-based cancer vaccine STINGVAX which consisted of CDNs and granulocyte-macrophage colony-stimulating factor (GM-CSF)-secreting cancer cells [61]. After a single dose of STINGVAX injection into the contralateral side of the transplanted B16 melanoma, tumor growth was significantly retarded in mice, and the treatment effect was dose-dependent [61]. Compared with GM-CSF-secreting cancer cell vaccine without formulation with CDNs, tumor tissues obtained from STINGVAX-treated mice had more infiltrating $\mathrm{CD}^{+} \mathrm{IFN}-\gamma^{+} \mathrm{T}$ cells [61]. In addition, the potent anti-cancer effect of STINGVAX was verified in multiple tumor-bearing mice models including colon cancer, digestive squamous cell cancer, and pancreatic cancer [61]. Further analysis showed that synthetic CDN had a strong immuno-stimulatory effect on both mouse and human DC [61]. These phenomena were later replicated in other studies, demonstrating the feasibility of using STING agonist as cancer vaccine adjuvant $[77,78]$.

\section{STING agonist: the sensitizer of ICI treatment}

ICI treatment mainly consists of anti-programmed death-1/programmed death-ligand 1 (PD-1/PD-L1) and anti-cytotoxic T-lymphocyte-associated protein 4 (CTLA-4) [79]. Anti-PD-1/PD-L1 monoclonal antibody $(\mathrm{mAb})$ restores TIL from exhausted status and enhances tumor-killing activity $[80,81]$. Anti-CTLA-4 mAb increases available co-stimulatory molecules (CD80 and CD86) and relieves competitive inhibition [82, 83]. Even though ICI treatment theoretically could reprogram tumor immune microenvironment and induce tumor regression, the actual clinical application is limited by low response rate.

\section{Anti-PD-1/PD-L1 combined with STING agonist}

STING agonist is the ideal sensitizer for anti-PD-1/ PD-L1 therapy. On the one hand, STING agonist enhances ICI treatment effect. Firstly, STING agonist promotes the infiltration of $\mathrm{T}$ cell into tumor. Pre-existing CTL is the precondition of robust anti-PD-1/PD-L1 treatment effect. Therefore, interventions promoting $\mathrm{T}$ cell infiltration into tumor is helpful to relieve anti-PD-1/PD-L1 resistance. Grabosch et al. used DNA-damage-inducing agent cisplatin to activate cGAS-STING pathway in mice bearing ovarian tumor [84]. The results showed that activated cytosolic DNA-sensor enhanced T cell infiltration [84]. This transformation to "hot tumor" might relate with the expression of IFN-stimulated genes (ISG) such as CXCL9 and CXCL10, which could recruit APC and T cell to tumor [85]. Secondly, cGAS-STING agonist counteracts the decrease of major histocompatibility complex (MHC) molecules on tumor cell which is an important approach to escape immune surveillance [86]. It was observed that cytosolic DNA-dependent IFN upregulation contributed to the increase of antigen presentation molecules (Tap1, Tap2, MHC I) [84]. Thirdly, cGAS-STING pathway elevates the sensitivity of tumor cell to immune killing activity of NK and CTL [50]. In the co-culture test, NK and CTL resistant tumor cell usually had higher NLRX1 and NLRC3 level, which antagonized the expression of cGAS-STING-induced type I IFN [50]. Actually, cGAS-STING pathway regulates anti-tumor immunity in a comprehensive manner from enhancing antigen presentation to increasing cytotoxicity. 
On the other hand, anti-PD-1/PD-L1 therapy neutralizes the immunosuppressive effect of cGAS-STING agonist [84]. It was reported that activated cGAS-STING accompanied the upregulation of PD-L1 expression [84]. By infecting PD-L1 ${ }^{\text {low }}$ mouse tumor cell (2F8 cell) with cGAS-STING-encoding adenovirus, nearly all infected tumor cell expressed PD-L1 while $46 \%$ of tumor cells infected by control adenovirus expressed PD-L1 [84]. Presumably, type I IFN also participates in the regulation of PD-L1 by phosphorylating JAK1-STAT1/STAT2/STAT3-IRF1 pathway, even in the less extent than IFN- $\gamma$ [87].

Tan et al. formulated nanosatellite vaccine SatVax with cGAMP and antigenic peptides (Q19D, Q15L) [50]. Combination therapy of SatVax plus anti-PD-L1 significantly increased E7-specific $\mathrm{CD}^{+}$CTL but simultaneously decreased the ratio of $\mathrm{CD}^{+} \mathrm{Tim}^{+}$and $\mathrm{CD} 8^{+} \mathrm{PD}-1^{+} \mathrm{T}$ cell in xenograft model [50]. The combination therapy induced potent tumor regression, and four of five mice achieved completely tumor-free status [50]. Similarly to the nanosatellite in SatVax, poly beta-amino ester (PBAE) nanoparticles could enhance the delivery of $\mathrm{CDN}$ as well [88]. In the mice bearing B16 melanoma, co-administration of PBAE-CDN and anti-PD-1 antibody markedly slowed tumor growth compared with anti-PD-1 plus unencapsulated CDN or anti-PD-1 monotherapy [88]. It was notable that mice that received STING agonist-combined anti-PD-1 treatment were resistant to tumor rechallenge in multiple xenograft models $[61,71]$. We proposed that cGAS-STING-induced type I IFN might promote the survival of memory tumor-specific CTLs.

\section{The role of CGAS-STING pathway in anti-CTLA-4 treatment}

Anti-CTLA-4 treatment reduces the activation threshold of $\mathrm{T}$ cells and magnifies the tumor-specific immune response $[89,90]$. Some studies revealed that anti-CTLA-4 mAb could selectively eradicate Tregs by antibody-dependent cell-mediated cytotoxicity (ADCC) effect [91, 92]. Shane et al. found intact cGAS-STING pathway was indispensable to maximized anti-CTLA-4 treatment effect [93]. Mice bearing B16 melanoma received the injection of irradiated tumor cells and subsequent anti-CTLA-4 treatment [93]. After combined treatment, no significant abscopal tumor eliminated effect was detected in mice receiving injection of STING-deficient B16 tumor cells [93]. In the meanwhile, STING deficiency markedly impaired $\mathrm{CD}^{+} \mathrm{T}$ infiltration in tumor bed [93].

Ager et al. investigated the efficacy of intratumoral injection of ICIs containing three checkpoint regulatory antibodies: anti-CTLA- 4 antibody (9H10), anti-PD-1 antibody (RMP114), and agonistic anti-4-1BB antibody (3H3) in mouse prostate cancer model [94]. The results showed that the ICI cocktail therapy eliminated bilateral tumors in $40 \%$ of mice while the contaminant administration of STING agonist CDG and triple immune checkpoint blockade induced bilateral tumor regression in $75 \%$ of mice [94]. By tracking the immunodominant neoepitope SPAS expressed in the established mouse prostate tumor, it was detected that the local administration of CDG and ICIs increased SPAS-specific $\mathrm{CD}^{+} \mathrm{T}$ cell in injected tumor. Further analysis revealed that the ratio of SPAS-specific $\mathrm{CD} 8^{+} \mathrm{T}$ cell to total tumor-infiltrating $\mathrm{CD}^{+} \mathrm{T}$ cell decreased [94]. Therefore, CDG combined ICIs effectively expanded T cell receptor (TCR) repertoire and activated immune response targeting subdominant antigens [94].

\section{The predictive role of CGAS-STING pathway in oncolytic virus}

As mentioned above, intact cGAS-STING pathway is the essential competent for host to defend the invasion of DNA viruses, retroviruses, and intracellular bacterial pathogens [95-97]. In the same time, it was found that multiple cancers harbored deficient cGAS-STING pathway such as colon cancer and melanoma [46, 98]. Thus, it is logical to choose oncolytic virus strategy for cGAS-STING-deficient cancer patients [98]. Xia et al. used herpes simplex virus type 1 (HSV-1) lacking $\gamma 34.5$ gene in mouse melanoma model [98]. The $\gamma 34.5$ viral protein could repress host innate immunity and HSV- $1 \Delta \gamma 34.5$ could effectively activate cGAS-STING pathway and clear virus infection in normal cells [98]. However, for cGAS-STING-deficient tumor cells, disabled anti-viral response resulted in rapid virus proliferation and ultimate cell death [98]. It was displayed that melanoma cells with deficient STING were susceptible to HSV- $1 \Delta \gamma 34.5$ infection [98]. In addition, Barber et al. found that cGAS-STING-deficient mice possessed higher response rate to intratumoral injection of HSV- $1 \Delta$ y34.5 in mouse ovarian cancer model [99]. Given the frequent inactivation of cGAS-STING in multiple cancers, usually caused by hypermethylation, it would be reasonable to apply oncolytic virus therapy in a subset of patients.

\section{Combined STING agonist and CAR-T therapy}

By transferring gene encoding CAR, engineered $T$ cell could specifically recognize target antigen on tumor cell with single-chain variable fragment (scFv) domain [100]. CAR-modified $\mathrm{T}$ cell is activated independent of MHC manner and then directly kills tumor cell [101, 102]. CAR-T therapy has been successfully applied in hematological diseases, but its effect is limited in solid tumors $[103,104]$. It is generally believed that immunosuppressive tumor microenvironment and intratumoral heterogeneity mainly contribute to escape from immune killing by CAR-T cell [105-107]. Smith et al. designed a novel implantable bioactive carrier which could deliver CAR-T cells to the surface of tumors [108]. Compared with systemic delivery of CAR-modified $\mathrm{T}$ cell, delivery by this bioactive carrier significantly enhanced $\mathrm{T}$ cell expansion and tumor control [108]. CAR-T therapy delivered by implanted scaffold prolonged survival time, but the 
intervention could not completely eliminate tumor in mice [108]. Under selective pressure, tumor cell with high expression of targeting expression (RAE1) were destroyed while RAE1 $1^{\text {low/negative }}$ tumor cells survived [108]. As a result, all mice developed resistance to CAR-T therapy [108]. Then, the bioactive scaffold was modified with additional STING agonist cyclic di-GMP (cdGMP) [108]. Co-delivery of cdGMP and CAR-T cells markedly increased the activation of downstream signaling pathway of the TCR/CD3 and circulating tumor-specific T cells [108]. In mouse pancreatic tumor model, combined delivery of CAR-T cells and cdGMP completely cleared tumor in four of ten mice and significantly prolonged survival time [108]. To further investigate this combination therapy induced systemic anti-tumor immunity, four mice undergoing complete regression were re-challenged with the intravenous injection of tumor cells [108]. It was notable that the prior combination treatment inhibited the formation of measurable tumor mass [108]. We proposed that STING agonist could boost the efficacy of CAR-T-induced in situ cancer vaccine and initiate durable systemic anti-tumor immune response.

\section{Conclusion}

Activated cGAS-STING pathway and its downstream signals boost the whole cancer-immunity cycle by enhancing cross-presentation and immune-killing activity. Therefore, cGAS-STING agonist is an ideal sensitizer for cancer immunotherapy and decreases the risk of drug resistance. On the one hand, STING agonist alters immune microenvironment from immunosuppressive type to immunosupportive type. On the other hand, as an identified adjuvant, STING agonist enhances the effect of treatment-induced in situ cancer vaccine and provides systemic memory anti-cancer effect. A growing body of evidence indicates that a cocktail of cGAS-STING agonist together with immunotherapy could effectively eradicate tumor mass and induce durable anti-tumor immune response. We believe manipulating cGAS-STING pathway might be a promising synergistic strategy with cancer immunotherapy.

\footnotetext{
Abbreviations

ADCC: Antibody dependent cell-mediated cytotoxicity; BAX: BCL2-associated X; CAR-T: Chimeric antigen receptor T cell; CdGMP: Cyclic di-GMP; CDN: Cyclic-dinucleotide; CGAMP: Cyclic GMP-AMP; CGAS: Cyclic GMP-AMP synthase; CTLA-4: Cytotoxic T-lymphocyte-associated protein 4; CXCL9: Chemokine (C-X-C motif) ligand 9; DC: Dendritic cell; DMXAA: Dimethyloxoxanthenyl acetic acid; dsDNA: Double-stranded DNA; EC50: Concentration for half maximal effect; ER: Endoplasmic reticulum; GMCSF: Granulocyte-macrophage colony-stimulating factor; HSV-1: Herpes simplex virus type 1; ICl: Immune checkpoint inhibitor; IDO: Indoleamine 2,3dioxygenase; IFN: Interferon; IKK: IKB kinase; IRF3: Interferon regulatory transcription factor 3; ISG: IFN-stimulated genes; mAb: Monoclonal antibody; ML: Mixed linkage; NF-KB: Nuclear factor kappa-light-chain-enhancer of activated B cell; NIK: NF-KB-inducing kinase; PBAE: Poly beta-amino ester; PD1: Programmed death-1; PD-L1: Programmed death-ligand 1; PRR: Pattern recognition receptor; ROS: Reactive oxygen species; scFv: Single-chain variable fragment; STING: Stimulator of interferon genes; TAA: Tumorassociated antigen; TBK1: TANK-binding kinase 1; TCR: T cell receptor; TDLN: Tumor-draining lymph node; Treg: Regulatory T cell
}

\section{Acknowledgements}

Not applicable.

\section{Funding}

This work was supported by the National Natural Science Foundation of China (No. 81874120, 81572608, 81672984), Wuhan Science and Technology Bureau (No. 2017060201010170).

\section{Availability of data and materials}

Data sharing not applicable to this article as no datasets were generated or analyzed during the current study.

\section{Authors' contributions}

AL performed the selection of literature. MY drafted the manuscript and prepared the figures. SQ, YS, and QC collected the related references and participated in the discussion. KW designed this review and revised the manuscript. All authors contributed to this manuscript. All authors read and approved the final manuscript.

Ethics approval and consent to participate

Not applicable.

Consent for publication

Not applicable.

Competing interests

The authors declare that they have no competing interests.

\section{Publisher's Note}

Springer Nature remains neutral with regard to jurisdictional claims in published maps and institutional affiliations.

\section{Author details \\ ${ }^{1}$ Department of Oncology, The First Affiliated Hospital of Zhengzhou University, Zhengzhou 450052, China. ${ }^{2}$ Department of Oncology, Tongji Hospital of Tongji Medical College, Huazhong University of Science and Technology, Wuhan 430030, China. ${ }^{3}$ Department of Hematology, the Affiliated Cancer Hospital of Zhengzhou University, Henan Cancer Hospital, Zhengzhou 450000, Henan, China.}

Received: 28 January 2019 Accepted: 11 March 2019

Published online: 01 April 2019

\section{References}

1. O'Donnell JS, Teng MWL, Smyth MJ. Cancer immunoediting and resistance to T cell-based immunotherapy. Nat Rev Clin Oncol. 2019;16:151-67.

2. Beatty GL, Gladney WL. Immune escape mechanisms as a guide for cancer immunotherapy. Clin Cancer Res. 2015;21:687-92.

3. Gajewski TF, Schreiber H, Fu YX. Innate and adaptive immune cells in the tumor microenvironment. Nat Immunol. 2013;14:1014-22.

4. Marin-Acevedo JA, Soyano AE, Dholaria B, Knutson KL, Lou Y. Cancer immunotherapy beyond immune checkpoint inhibitors. J Hematol Oncol. 2018;11:8.

5. Yang Y. Cancer immunotherapy: harnessing the immune system to battle cancer. J Clin Invest. 2015;125:3335-7.

6. Chen DS, Mellman I. Oncology meets immunology: the cancer-immunity cycle. Immunity. 2013;39:1-10.

7. Yu S, Li A, Liu Q, Li T, Yuan X, Han X, et al. Chimeric antigen receptor T cells: a novel therapy for solid tumors. J Hematol Oncol. 2017;10:78.

8. Yu S, Liu Q, Han X, Qin S, Zhao W, Li A, et al. Development and clinical application of anti-HER2 monoclonal and bispecific antibodies for cancer treatment. Exp Hematol Oncol. 2017;6:31.

9. Fukuhara $H$, Ino $Y$, Todo T. Oncolytic virus therapy: a new era of cancer treatment at dawn. Cancer Sci. 2016;107:1373-9.

10. Yi M, Jiao D, Xu H, Liu Q, Zhao W, Han X, et al. Biomarkers for predicting efficacy of PD-1/PD-L1 inhibitors. Mol Cancer. 2018;17:129.

11. Sayour EJ, Mitchell DA. Manipulation of innate and adaptive immunity through cancer vaccines. J Immunol Res. 2017;2017:3145742.

12. Thaiss CA, Zmora N, Levy M, Elinav E. The microbiome and innate immunity. Nature. 2016;535:65-74. 
13. Khoo LT, Chen LY. Role of the CGAS-STING pathway in cancer development and oncotherapeutic approaches. EMBO Rep. 2018. https://doi.org/10. 15252/embr.201846935.

14. Chen Q, Sun L, Chen ZJ. Regulation and function of the CGAS-STING pathway of cytosolic DNA sensing. Nat Immunol. 2016;17:1142-9.

15. Margolis SR, Wilson SC, Vance RE. Evolutionary origins of CGAS-STING signaling. Trends Immunol. 2017;38:733-43.

16. Ma Z, Damania B. The CGAS-STING defense pathway and its counteraction by viruses. Cell Host Microbe. 2016;19:150-8.

17. Gajewski TF, Corrales L. New perspectives on type I IFNs in cancer. Cytokine Growth Factor Rev. 2015;26:175-8.

18. Fuertes MB, Woo SR, Burnett B, Fu YX, Gajewski TF. Type I interferon response and innate immune sensing of cancer. Trends Immunol. 2013;34:67-73.

19. Ishikawa H, Barber GN. STING is an endoplasmic reticulum adaptor that facilitates innate immune signalling. Nature. 2008;455:674-8.

20. Zhong B, Yang Y, Li S, Wang YY, Li Y, Diao F, et al. The adaptor protein MITA links virus-sensing receptors to IRF3 transcription factor activation. Immunity. 2008;29:538-50.

21. Luo M, Wang H, Wang Z, Cai H, Lu Z, Li Y, et al. A STING-activating nanovaccine for cancer immunotherapy. Nat Nanotechnol. 2017;12:648-54

22. Chandra D, Quispe-Tintaya W, Jahangir A, Asafu-Adjei D, Ramos I, Sintim $\mathrm{HO}$, et al. STING ligand c-di-GMP improves cancer vaccination against metastatic breast cancer. Cancer Immunol Res. 2014;2:901-10.

23. Moore E, Clavijo PE, Davis R, Cash H, Van Waes C, Kim Y, et al. Established T cell-inflamed tumors rejected after adaptive resistance was reversed by combination STING activation and PD-1 pathway blockade. Cancer Immunol Res. 2016:4:1061-71.

24. Temizoz B, Kuroda E, Ishii KJ. Combination and inducible adjuvants targeting nucleic acid sensors. Curr Opin Pharmacol. 2018;41:104-13.

25. Corrales L, McWhirter SM, Dubensky TW Jr, Gajewski TF. The host STING pathway at the interface of cancer and immunity. J Clin Invest. 2016;126:2404-11.

26. Burdette $\mathrm{DL}$, Vance RE. STING and the innate immune response to nucleic acids in the cytosol. Nat Immunol. 2013;14:19-26.

27. Ishikawa H, Ma Z, Barber GN. STING regulates intracellular DNA-mediated, type I interferon-dependent innate immunity. Nature. 2009;461:788-92.

28. Burdette DL, Monroe KM, Sotelo-Troha K, Iwig JS, Eckert B, Hyodo M, et al. STING is a direct innate immune sensor of cyclic di-GMP. Nature. 2011;478: 515-8.

29. Gao P, Ascano M, Wu Y, Barchet W, Gaffney BL, Zillinger T, et al. Cyclic $\left[G\left(2^{\prime}, 5^{\prime}\right) p A\left(3^{\prime}, 5^{\prime}\right) p\right]$ is the metazoan second messenger produced by DNAactivated cyclic GMP-AMP synthase. Cell. 2013;153:1094-107.

30. Ablasser A, Goldeck M, Cavlar T, Deimling T, Witte G, Rohl I, et al. cGAS produces a 2'-5'-linked cyclic dinucleotide second messenger that activates STING. Nature. 2013;498:380-4.

31. Liu S, Cai X, Wu J, Cong Q, Chen X, Li T, et al. Phosphorylation of innate immune adaptor proteins MAVS, STING, and TRIF induces IRF3 activation. Science. 2015;347:aaa2630

32. Barber GN. STING: infection, inflammation and cancer. Nat Rev Immunol 2015:15:760-70

33. Abe T, Barber GN. Cytosolic-DNA-mediated, STING-dependent proinflammatory gene induction necessitates canonical NF-kappaB activation through TBK1. J Virol. 2014;88:5328-41.

34. Ablasser A, Schmid-Burgk JL, Hemmerling I, Horvath GL, Schmidt T, Latz E, et al. Cell intrinsic immunity spreads to bystander cells via the intercellular transfer of cGAMP. Nature. 2013;503:530-4.

35. Liu H, Zhang H, Wu X, Ma D, Wu J, Wang L, et al. Nuclear cGAS suppresses DNA repair and promotes tumorigenesis. Nature. 2018;563:131-6.

36. Roers A, Hiller B, Hornung $V$. Recognition of endogenous nucleic acids by the innate immune system. Immunity. 2016;44:739-54.

37. Ho SS, Zhang WY, Tan NY, Khatoo M, Suter MA, Tripathi S, et al. The DNA structure-specific endonuclease MUS81 mediates DNA sensor STING dependent host rejection of prostate cancer cells. Immunity. 2016;44:1177-89.

38. Shen YJ, Le Bert N, Chitre AA, Koo CX, Nga XH, Ho SS, et al. Genomederived cytosolic DNA mediates type I interferon-dependent rejection of $B$ cell lymphoma cells. Cell Rep. 2015;11:460-73.

39. Lam AR, Bert NL, Ho SS, Shen YJ, Tang LF, Xiong GM, et al. RAE1 ligands for the NKG2D receptor are regulated by STING-dependent DNA sensor pathways in lymphoma. Cancer Res. 2014;74:2193-203.

40. Mackenzie KJ, Carroll P, Martin CA, Murina O, Fluteau A, Simpson DJ, et al. cGAS surveillance of micronuclei links genome instability to innate immunity. Nature. 2017;548:461-5.
41. Gisselsson D, Bjork J, Hoglund M, Mertens F, Dal Cin P, Akerman M, et al Abnormal nuclear shape in solid tumors reflects mitotic instability. Am J Pathol. 2001;158:199-206.

42. Santaguida S, Richardson A, lyer DR, M'Saad O, Zasadil L, Knouse KA, et al. Chromosome mis-segregation generates cell-cycle-arrested cells with complex karyotypes that are eliminated by the immune system. Dev Cell. 2017:41:638-51 e5.

43. Hintzsche H, Hemmann U, Poth A, Utesch D, Lott J, Stopper H. Fate of micronuclei and micronucleated cells. Mutat Res. 2017;771:85-98.

44. Zhang CZ, Spektor A, Cornils H, Francis JM, Jackson EK, Liu S, et al. Chromothripsis from DNA damage in micronuclei. Nature. 2015;522:179-84.

45. Ahn J, Xia T, Konno H, Konno K, Ruiz P, Barber GN. Inflammation-driven carcinogenesis is mediated through STING. Nat Commun. 2014;5:5166.

46. Xia T, Konno H, Ahn J, Barber GN. Deregulation of STING signaling in colorectal carcinoma constrains DNA damage responses and correlates with tumorigenesis. Cell Rep. 2016;14:282-97.

47. Ahn J, Konno H, Barber GN. Diverse roles of STING-dependent signaling on the development of cancer. Oncogene. 2015;34:5302-8.

48. Liu Y, Dong Y, Kong L, Shi F, Zhu H, Yu J. Abscopal effect of radiotherapy combined with immune checkpoint inhibitors. J Hematol Oncol. 2018;11:104

49. Carroll EC, Jin L, Mori A, Munoz-Wolf N, Oleszycka E, Moran HBT, et al. The vaccine adjuvant chitosan promotes cellular immunity via DNA sensor cGAS-STING-dependent induction of type I interferons. Immunity. 2016:44: 597-608.

50. Tan YS, Sansanaphongpricha K, Xie Y, Donnelly CR, Luo X, Heath BR, et al. Mitigating SOX2-potentiated immune escape of head and neck squamous cell carcinoma with a STING-inducing nanosatellite vaccine. Clin Cancer Res. 2018:24:4242-55.

51. Hsu YA, Huang CC, Kung YJ, Lin HJ, Chang CY, Lee KR, et al. The antiproliferative effects of type I IFN involve STAT6-mediated regulation of SP1 and BCL6. Cancer Lett. 2016;375:303-12.

52. Vanpouille-Box C, Demaria S, Formenti SC, Galluzzi L. Cytosolic DNA sensing in organismal tumor control. Cancer Cell. 2018;34:361-78.

53. Chattopadhyay S, Marques JT, Yamashita M, Peters KL, Smith K, Desai A, et al. Viral apoptosis is induced by IRF-3-mediated activation of Bax. EMBO J. 2010:29:1762-73.

54. Woo SR, Fuertes MB, Corrales L, Spranger S, Furdyna MJ, Leung MY, et al. STING-dependent cytosolic DNA sensing mediates innate immune recognition of immunogenic tumors. Immunity. 2014;41:830-42.

55. Parlato S, Santini SM, Lapenta C, Di Pucchio T, Logozzi M, Spada M, et al. Expression of CCR-7, MIP-3beta, and Th-1 chemokines in type I IFN-induced monocyte-derived dendritic cells: importance for the rapid acquisition of potent migratory and functional activities. Blood. 2001;98:3022-9.

56. Peng D, Kryczek I, Nagarsheth N, Zhao L, Wei S, Wang W, et al. Epigenetic silencing of TH1-type chemokines shapes tumour immunity and immunotherapy. Nature. 2015;527:249-53.

57. Deng L, Liang $H$, Xu M, Yang X, Burnette B, Arina A, et al. STING-dependent cytosolic DNA sensing promotes radiation-induced type I interferondependent antitumor immunity in immunogenic tumors. Immunity. 2014; 41:843-52.

58. Larkin B, Ilyukha V, Sorokin M, Buzdin A, Vannier E, Poltorak A. Cutting edge: activation of STING in T cells induces type I IFN responses and cell death. J Immunol. 2017:199:397-402.

59. Cerboni S, Jeremiah N, Gentili M, Gehrmann U, Conrad C, Stolzenberg MC, et al. Intrinsic antiproliferative activity of the innate sensor STING in T lymphocytes. J Exp Med. 2017;214:1769-85.

60. Sivick KE, Desbien AL, Glickman LH, Reiner GL, Corrales L, Surh NH, et al. Magnitude of therapeutic STING activation determines CD8(+) T cellmediated anti-tumor immunity. Cell Rep. 2018;25:3074-85 e5.

61. Fu J, Kanne DB, Leong M, Glickman LH, McWhirter SM, Lemmens E, et al. STING agonist formulated cancer vaccines can cure established tumors resistant to PD-1 blockade. Sci Transl Med. 2015;7:283ra52.

62. Klarquist J, Hennies CM, Lehn MA, Reboulet RA, Feau S, Janssen EM. STINGmediated DNA sensing promotes antitumor and autoimmune responses to dying cells. J Immunol. 2014;193:6124-34.

63. Marcus A, Mao AJ, Lensink-Vasan M, Wang L, Vance RE, Raulet DH. Tumorderived CGAMP triggers a STING-mediated interferon response in nontumor cells to activate the NK cell response. Immunity. 2018;49:754-63 e4.

64. Lemos H, Mohamed E, Huang L, Ou R, Pacholczyk G, Arbab AS, et al. STING promotes the growth of tumors characterized by low antigenicity via IDO activation. Cancer Res. 2016;76:2076-81. 
65. Liu M, Wang X, Wang L, Ma X, Gong Z, Zhang S, et al. Targeting the IDO1 pathway in cancer: from bench to bedside. J Hematol Oncol. 2018;11:100.

66. Liang D, Xiao-Feng H, Guan-Jun D, Er-Ling H, Sheng C, Ting-Ting W, et al. Activated STING enhances Tregs infiltration in the HPV-related carcinogenesis of tongue squamous cells via the c-jun/CCL22 signal. Biochim Biophys Acta. 1852;2015:2494-503.

67. Mukai K, Konno H, Akiba T, Uemura T, Waguri S, Kobayashi T, et al. Activation of STING requires palmitoylation at the Golgi. Nat Commun. 2016;7:11932.

68. Conlon J, Burdette DL, Sharma S, Bhat N, Thompson M, Jiang Z, et al. Mouse, but not human STING, binds and signals in response to the vascular disrupting agent 5,6-dimethylxanthenone-4-acetic acid. J Immunol. 2013; 190:5216-25.

69. Corrales L, Glickman LH, McWhirter SM, Kanne DB, Sivick KE, Katibah GE, et al. Direct activation of STING in the tumor microenvironment leads to potent and systemic tumor regression and immunity. Cell Rep. 2015;11: 1018-30.

70. Foote JB, Kok M, Leatherman JM, Armstrong TD, Marcinkowski BC, Ojalvo LS, et al. A STING agonist given with OX40 receptor and PD-L1 modulators primes immunity and reduces tumor growth in tolerized mice. Cancer Immunol Res. 2017;5:468-79.

71. Shae D, Becker KW, Christov P, Yun DS, Lytton-Jean AKR, Sevimli S, et al. Endosomolytic polymersomes increase the activity of cyclic dinucleotide STING agonists to enhance cancer immunotherapy. Nat Nanotechnol. 2019; 14:269-78.

72. Ramanjulu JM, Pesiridis GS, Yang J, Concha N, Singhaus R, Zhang SY, et al. Design of amidobenzimidazole STING receptor agonists with systemic activity. Nature. 2018;564:439-43.

73. Dubensky TW Jr, Reed SG. Adjuvants for cancer vaccines. Semin Immunol. 2010;22:155-61.

74. Yi M, Qin S, Zhao W, Yu S, Chu Q, Wu K. The role of neoantigen in immune checkpoint blockade therapy. Exp Hematol Oncol. 2018;7:28.

75. Alvarez-Arguedas S, Uranga S, Martin M, Elizalde J, Gomez AB, Julian E, et al. Therapeutic efficacy of the live-attenuated Mycobacterium tuberculosis vaccine, MTBVAC, in a preclinical model of bladder cancer. Transl Res. 2018; 197:32-42.

76. Madhun AS, Haaheim LR, Nostbakken JK, Ebensen T, Chichester J, Yusibov V, et al. Intranasal c-di-GMP-adjuvanted plant-derived $\mathrm{H} 5$ influenza vaccine induces multifunctional Th1 CD4+ cells and strong mucosal and systemic antibody responses in mice. Vaccine. 2011;29:4973-82.

77. Wang Z, Celis E. STING activator c-di-GMP enhances the anti-tumor effects of peptide vaccines in melanoma-bearing mice. Cancer Immunol Immunother. 2015;64:1057-66.

78. Kinkead HL, Hopkins A, Lutz E, Wu AA, Yarchoan M, Cruz K, et al. Combining STING-based neoantigen-targeted vaccine with checkpoint modulators enhances antitumor immunity in murine pancreatic cancer. JCI Insight. 2018. https://doi.org/10.1172/jci.insight.122857.

79. Li X, Shao C, Shi Y, Han W. Lessons learned from the blockade of immune checkpoints in cancer immunotherapy. J Hematol Oncol. 2018;11:31.

80. Sacher AG, Gandhi L. Biomarkers for the clinical use of PD-1/PD-L1 inhibitors in non-small-cell lung cancer: a review. JAMA Oncol. 2016;2:1217-22.

81. Marin-Acevedo JA, Dholaria B, Soyano AE, Knutson KL, Chumsri S, Lou Y. Next generation of immune checkpoint therapy in cancer: new developments and challenges. J Hematol Oncol. 2018;11:39.

82. Lipson EJ, Drake CG. Ipilimumab: an anti-CTLA-4 antibody for metastatic melanoma. Clin Cancer Res. 2011;17:6958-62.

83. Yi M, Yu S, Qin S, Liu Q, Xu H, Zhao W, et al. Gut microbiome modulates efficacy of immune checkpoint inhibitors. J Hematol Oncol. 2018;11:47.

84. Grabosch S, Bulatovic M, Zeng F, Ma T, Zhang L, Ross M, et al. Cisplatin-induced immune modulation in ovarian cancer mouse models with distinct inflammation profiles. Oncogene. 2018. https://doi.org/10.1038/s41388-018-0581-9.

85. Corbera-Bellalta M, Planas-Rigol E, Lozano E, Terrades-Garcia N, Alba MA, Prieto-Gonzalez S, et al. Blocking interferon gamma reduces expression of chemokines CXCL9, CXCL10 and CXCL11 and decreases macrophage infiltration in ex vivo cultured arteries from patients with giant cell arteritis. Ann Rheum Dis. 2016;75:1177-86

86. Reeves $E$, James $E$. Antigen processing and immune regulation in the response to tumours. Immunology. 2017;150:16-24.

87. Garcia-Diaz A, Shin DS, Moreno BH, Saco J, Escuin-Ordinas H, Rodriguez GA, et al. Interferon receptor signaling pathways regulating PD-L1 and PD-L2 expression. Cell Rep. 2017;19:1189-201.
88. Wilson DR, Sen R, Sunshine JC, Pardoll DM, Green JJ, Kim YJ. Biodegradable STING agonist nanoparticles for enhanced cancer immunotherapy. Nanomedicine. 2018;14:237-46.

89. Liu J, Yang Y, Wang H, Wang B, Zhao K, Jiang W, et al. Syntenin1/MDA-9 (SDCBP) induces immune evasion in triple-negative breast cancer by upregulating PD-L1. Breast Cancer Res Treat. 2018;171:345-57.

90. Ok CY, Young KH. Checkpoint inhibitors in hematological malignancies. J Hematol Oncol. 2017:10:103.

91. Arce Vargas F, Furness AJS, Litchfield K, Joshi K, Rosenthal R, Ghorani E, et al. Fc effector function contributes to the activity of human anti-CTLA-4 antibodies. Cancer Cell. 2018;33:649-63 e4.

92. Ingram JR, Blomberg OS, Rashidian M, Ali L, Garforth S, Fedorov E, et al. Anti-CTLA-4 therapy requires an Fc domain for efficacy. Proc Natl Acad Sci U S A. 2018;115:3912-7.

93. Harding SM, Benci JL, Irianto J, Discher DE, Minn AJ, Greenberg RA. Mitotic progression following DNA damage enables pattern recognition within micronuclei. Nature. 2017:548:466-70.

94. Ager CR, Reilley MJ, Nicholas C, Bartkowiak T, Jaiswal AR, Curran MA. Intratumoral STING activation with T-cell checkpoint modulation generates systemic antitumor immunity. Cancer Immunol Res. 2017;5:676-84.

95. Gao D, Wu J, Wu YT, Du F, Aroh C, Yan N, et al. Cyclic GMP-AMP synthase is an innate immune sensor of HIV and other retroviruses. Science. 2013;341:903-6.

96. Storek KM, Gertsvolf NA, Ohlson MB, Monack DM. cGAS and Ifi204 cooperate to produce type I IFNs in response to Francisella infection. J Immunol. 2015;194:3236-45.

97. Schoggins JW, MacDuff DA, Imanaka N, Gainey MD, Shrestha B, Eitson JL, et al. Pan-viral specificity of IFN-induced genes reveals new roles for CGAS in innate immunity. Nature. 2014;505:691-5.

98. Xia T, Konno H, Barber GN. Recurrent loss of STING signaling in melanoma correlates with susceptibility to viral oncolysis. Cancer Res. 2016;76:6747-59.

99. Barber GN, Gual Pimenta de Queiroz NM, Xia T, Konno H. Ovarian cancer cells commonly exhibit defective STING signaling which affects sensitivity to viral oncolysis. Mol Cancer Res. 2018; doi: https://doi.org/10.1158/1541-7786. MCR-18-0504.

100. Eshhar Z, Waks T, Gross G, Schindler DG. Specific activation and targeting of cytotoxic lymphocytes through chimeric single chains consisting of antibody-binding domains and the gamma or zeta subunits of the immunoglobulin and T-cell receptors. Proc Natl Acad Sci U S A. 1993;90: $720-4$.

101. Wang Z, Wu Z, Liu Y, Han W. New development in CAR-T cell therapy. J Hematol Oncol. 2017;10:53.

102. Qin L, Zhao R, Li P. Incorporation of functional elements enhances the antitumor capacity of CAR T cells. Exp Hematol Oncol. 2017;6:28.

103. Scarfo I, Maus MV. Current approaches to increase CAR T cell potency in solid tumors: targeting the tumor microenvironment. J Immunother Cancer. 2017:5:28.

104. Pang Y, Hou X, Yang C, Liu Y, Jiang G. Advances on chimeric antigen receptor-modified T-cell therapy for oncotherapy. Mol Cancer. 2018;17:91.

105. Newick K, O'Brien S, Moon E, Albelda SM. CAR T cell therapy for solid tumors. Annu Rev Med. 2017;68:139-52.

106. Zhang E, Gu J, Xu H. Prospects for chimeric antigen receptor-modified T cell therapy for solid tumors. Mol Cancer. 2018;17:7.

107. Li J, Li W, Huang K, Zhang Y, Kupfer G, Zhao Q. Chimeric antigen receptor T cell (CAR-T) immunotherapy for solid tumors: lessons learned and strategies for moving forward. J Hematol Oncol. 2018;11:22.

108. Smith TT, Moffett HF, Stephan SB, Opel CF, Dumigan AG, Jiang X, et al. Biopolymers codelivering engineered T cells and STING agonists can eliminate heterogeneous tumors. J Clin Invest. 2017;127:2176-91.

109. Tang $\mathrm{CH}$, Zundell JA, Ranatunga $\mathrm{S}$, Lin $\mathrm{C}$, Nefedova $Y$, Del Valle JR, et al. Agonist-mediated activation of STING induces apoptosis in malignant B cells. Cancer Res. 2016;76:2137-52.

110. Jassar AS, Suzuki E, Kapoor V, Sun J, Silverberg MB, Cheung L, et al. Activation of tumor-associated macrophages by the vascular disrupting agent 5,6-dimethylxanthenone-4-acetic acid induces an effective CD8+ Tcell-mediated antitumor immune response in murine models of lung cancer and mesothelioma. Cancer Res. 2005:65:11752-61.

111. McKeage MJ, Reck M, Jameson MB, Rosenthal MA, Gibbs D, Mainwaring PN, et al. Phase II study of ASA404 (vadimezan, 5,6-dimethylxanthenone-4-acetic acid/DMXAA) $1800 \mathrm{mg} / \mathrm{m}$ (2) combined with carboplatin and paclitaxel in previously untreated advanced non-small cell lung cancer. Lung Cancer. 2009:65:192-7. 
112. Pili R, Rosenthal MA, Mainwaring PN, Van Hazel G, Srinivas S, Dreicer R, et al. Phase II study on the addition of ASA404 (vadimezan; 5,6-

dimethylxanthenone-4-acetic acid) to docetaxel in CRMPC. Clin Cancer Res. 2010;16:2906-14.

113. Nakamura T, Miyabe H, Hyodo M, Sato $Y$, Hayakawa $Y$, Harashima $H$. Liposomes loaded with a STING pathway ligand, cyclic di-GMP, enhance cancer immunotherapy against metastatic melanoma. J Control Release. 2015;216:149-57.

114. Ohkuri T, Ghosh A, Kosaka A, Zhu J, Ikeura M, David M, et al. STING contributes to antiglioma immunity via triggering type I IFN signals in the tumor microenvironment. Cancer Immunol Res. 2014;2:1199-208.

Ready to submit your research? Choose BMC and benefit from:

- fast, convenient online submission

- thorough peer review by experienced researchers in your field

- rapid publication on acceptance

- support for research data, including large and complex data types

- gold Open Access which fosters wider collaboration and increased citations

- maximum visibility for your research: over $100 \mathrm{M}$ website views per year

At $\mathrm{BMC}$, research is always in progress.

Learn more biomedcentral.com/submissions 